\title{
PREDIÇÃO IN SILICO DE MARCADORES MICROSSATÉLITES RELACIONADOS AO TEGUMENTO DE SEMENTES DE SOJA ${ }^{1}$
}

\author{
FERNANDO AUGUSTO HENINNG ${ }^{2}$, LUCIANO CARLOS DA MAIA ${ }^{3}$, LILIANE MARCIA MERTZ², \\ PAULO DEJALMA ZIMMER ${ }^{4}$, ANTÔNIO COSTA DE OLIVEIRA ${ }^{5}$
}

\begin{abstract}
RESUMO - O sucesso na obtenção de altas produtividades de soja depende da utilização de sementes de qualidade. Muitos problemas que comprometem a qualidade fisiológica das sementes podem ser relacionados às características do tegumento. Inúmeros trabalhos afirmam que sementes de soja com tegumento semipermeável à água apresentam maior tolerância a patógenos e pragas, menor susceptibilidade aos danos mecânicos, às adversidades climáticas e à deterioração por umidade. A inclusão das características de tegumento semipermeável nas cultivares atuais pode minimizar problemas relacionados à qualidade de sementes. Neste contexto, aliar as técnicas da biologia molecular com a bioinformática é uma importante estratégia para identificação dos genes envolvidos com o tegumento e com a fisiologia de sementes. O objetivo desse trabalho foi descrever e avaliar uma estratégia de utilização de ferramentas da bioinformática, para a integração in silico de informações de experimentos in vitro de marcadores moleculares, contra dados armazenados em bancos de dados genômicos e prever pela descrição funcional se estes marcadores podem estar associados a diferentes características do tegumento da soja. Foram utilizados 24 conjuntos de primers microssatélites, avaliados anteriormente e que amplificaram fragmentos polimórficos entre os genótipos de soja CD202 (tegumento amarelo, permeável e susceptível à deterioração) e uma linhagem TP (tegumento preto, semipermeável e resistente a deterioração). Os resultados desta análise indicam como promissor o uso destes marcadores para estudos relacionados ao tegumento e à qualidade de sementes de soja. A estratégia da mineração de marcadores moleculares a partir da integração in silico de sequências de marcadores moleculares ainda anônimos, bancos de dados genômicos e bancos contendo seqüências com descrições funcionais dos genes demonstra ser promissora, pois, possibilita prever as funções para estes genes e verificar a associação destes com rotas bioquímicas e metabólicas responsáveis pelas características que se deseja analisar em rotinas in vitro.
\end{abstract}

Termos para indexação: Bioinformática, Marcadores Moleculares, Glycine max.

\section{IN SILICO PREDICTION OF MICROSATELLITE MARKERS RELATED TO SOYBEAN SEED COAT}

\begin{abstract}
Success in obtaining high soybean productivities depends on the use of high. quality seed. Many problems that damage the seed physiological quality can be related to the seed coat traits. Several studies state the existence of soybean seeds with a semi-permeable coat, which are more resistant to water penetration and less susceptible to mechanical damage, climatic adversities
\end{abstract}

${ }^{1}$ Submetido para publicação em 27/10/2008. Aceito para publicação $16 / 06 / 2009$

${ }^{2}$ Eng. Agr.,- MSc. - Discente Pós-Graduação em Ciência e Tecnologia de Sementes - FAEM/UFPel. fernandohenning@yahoo.com.br

${ }^{3}$ Eng. Agr.,- MSc. - Discente Pós-Graduação em Agronomia, área de concentração Fitomelhoramento - FAEM/UFPel.

${ }^{4}$ Eng. Agr., Dr. , Professor do Departamento de Fitotecnia - Ciência e Tecnologia de sementes - FAEM/UFPel. Cep: 96010-97 - Pelotas-RS

${ }^{5}$ Eng. Agr., Dr., Professor do Departamento de Fitotecnia - Centro de Genômica e Fitomelhoramento - FAEM/UFPel. 
and moisture deterioration. The inclusion of the traits for semi-permeable coat in modern cultivars could minimize problems related to seed quality. In this context, molecular biology techniques in association with bioinformatics are an important alternative to identify genes involved in soybean seed coat and physiological quality. The objective of this study was to describe and to evaluate a strategy using bioinformatics tools, to integrate in silico information with in vitro experiments of molecular markers against data available in genomic databases, and provide information as to whether these markers could be associated with different soybean seed coat traits. Twenty four microssatelite primer sets previously tested and which amplified polymorphic fragments between soybean genotypes CD-202 (yellow seed coat, permeable and susceptible to deterioration) and TP (black seed coat, semi-permeable and resistant to deterioration) were used. The results indicated as promising the use of these molecular markers for studies related to soybean seed coat and quality. The strategy of molecular markers mining from in silico integration of yet anonymous molecular marker sequences and database containing gene sequences with functional descriptions, seems to be promising, as it allowed to predict the functions of these genes and to verify their association with metabolic and biochemical pathways involved in characteristics which are interesting to in vitro analyzes.

Index terms: Bioinformatics, Molecular Markers, Glycine max.

\section{INTRODUÇÃO}

A absorção de umidade pela semente de soja quando esta ainda encontra-se no campo em períodos que antecedem a colheita, pode ser apontada como a principal causa para a baixa qualidade, direcionando a atenção de pesquisadores para sementes com tegumento semipermeável. O tegumento semipermeável é uma característica muito importante no que concerne à deterioração em campo, pois ao controlar melhor a absorção de água, as sementes se tornam mais resistentes às condições adversas (Peske e Pereira, 1983).

A característica tegumento semipermeável pode ser utilizada nos programas de melhoramento de soja para ser incorporada às cultivares de alta produtividade, visando à qualidade fisiológica da semente. Nesse sentido, ferramentas da biologia molecular aliada a bioinformática podem auxiliar na caracterização de genes ou regiões genômicas envolvidas no controle deste caráter e suas respostas fisiológicas nas sementes.

Microssatélites ou SSRs (Simple sequence repeats) (Litt e Lutty, 1989; Tautz et al., 1989) são sequências de nucleotídeos constituídas por arranjos formados por 1, 2, 3, 4, 5 ou 6 nucleotídeos que repetem lado a lado (em série), encontrados em alta frequência nos genomas de procariotos e eucariotos (Morgante e Oliveira, 1993). No passado, a grande limitação para o uso da técnica dos microssatélites em larga escala era a obtenção dos primers usados na PCR (Polimerase Chain Reaction), sendo uma estratégia trabalhosa e demorada, pois em geral, somente 10 a $20 \%$ dos primers desenvolvidos pelos protocolos tradicionais são informativos ou eficientes em estudos genéticos de uma população, contribuindo, desta forma, para a elevação dos custos e envolvimento de pessoal especializado (Buso et al., 2003).

A partir de meados dos anos 90, projetos de sequenciamento de genomas completos ou ainda projetos de sequenciamento de regiões funcionais de varias espécies, incluindo as espécies vegetais de maior importância agronômica, tem disponibilizado um grande número de seqüências em bancos de dados como o NCBI (National Center for Biotechnology Information), EMBL (European Molecular Biology Laboratory), GRAMENE ( $A$ Comparative Mapping Resource for Grains) e TIGR (The Institute for Genomic Research) entre outros. Nesse sentido, a localização e/ou prospecção de genes é possibilitada através da utilização de técnicas da biologia molecular e de ferramentas de bioinformática.

Atualmente, com o grande número de informações disponibilizadas nos bancos de DNA, a busca de marcadores moleculares nestes bancos é uma estratégia que minimiza as dificuldades encontradas no passado. Além disso, a disponibilidade dessas informações permite através do uso de ferramentas adequadas, a busca por homologia entre a sequência acessada por um determinado marcador com genes ou proteínas de função descrita, possibilitando predizer se estes marcadores acessam genes envolvidos nas 
rotas metabólicas do caráter em estudo.

No portal GRAMENE(Liang et al., 2007) estão indexados diversos mapas genéticos e marcadores moleculares para gramíneas, onde a estratégia da comparação por homologia foi utilizada para descrever e /ou predizer funções gênicas para marcadores baseados em sequências de sondas RFLP (Restriction Fragment Length Polymorphism), seqüências de cDNA (Complementary DNA) e SSRs. Neste banco de dados as sequências RFLP, marcadores baseados em cDNA e SSRs estão associadas pelo programa BLAST (Altschul et al., 1990) a outros bancos de dados contendo genes descritos e demais informações genômicas.

No banco de dados conhecido como GRAINGENES (Matthews et al. 2003) desenvolvido pelo Departamento de Agricultura dos Estados Unidos, para centralizar informações para o melhoramento genético de trigo, cevada, centeio, triticale e aveia também foi utilizada a mesma estratégia, onde, sequências de marcadores RFLP, SSRs e demais marcadores associados a QTLs (Quantitative Trait Loci) de 81 mapas genéticos foram associados a informações genômicas de outros bancos, sendo que cada um dos marcadores armazenados naquele banco, contem uma possível descrição funcional baseada no alinhamento feito pelo programa BLAST, contra demais bancos de dados. Poncet et al. (2005) utilizou a estratégia do alinhamento contra vários genomas para descrever as possíveis funções gênicas para 23 marcadores CAPS (Cleaved Amplified Polymorfic Sequences) derivados de fragmentos AFLP (Amplified Fragment Length Polymorphism) extraídos de um mapeamento genético de híbridos de Coffea sp.

O objetivo desse trabalho foi descrever e avaliar uma estratégia de utilização de ferramentas da bioinformática, para a integração in silico de informações de experimentos in vitro de marcadores moleculares contra dados armazenadas em bancos de dados genômicos, e prever pela descrição funcional se estes marcadores podem estar associados a diferentes características do tegumento da soja.

\section{MATERIAL E MÉTODOS}

\section{Localização dos primers e sequências genômicas:}

Neste estudo foram utilizadas as sequências amplificadas pelos 24 conjuntos de primers microssatélites, avaliados anteriormente e que amplificaram fragmentos polimórficos entre os genótipos de soja CD-202 (tegumento amarelo, permeável e susceptível a deterioração) e uma linhagem TP (tegumento preto, semipermeável e resistente a deterioração) (Henning, 2007).
A partir do banco de dados SoyBase (http://soybase. agron.iastate.edu) foram encontrados informações da nomenclatura dos 24 conjuntos de primers, posteriormente, as sequências amplificadas por estes conjuntos de primers foram encontradas no NCBI. Estas sequências foram utilizadas como molde (sequências de busca) para as buscas por similaridades no NCBI, utilizando a estratégia do alinhamento local através do programa computacional BLAST, adotando-se os parâmetros de configuração padrão do programa.

Foram considerados como homologias, os alinhamentos com similaridades baseada na estatística $E$-value, e que apresentaram valores inferiores a $1 \mathrm{e}^{-10}$. O valor do E-value estima a probabilidade de que o alinhamento entre sequências tenha ocorrido ao acaso.

\section{RESULTADOS E DISCUSSÃO}

Entre os 24 conjuntos de primers analisados, as sequências amplificadas (amplicons) por 15 conjuntos apresentaram homologias somente com seqüências derivadas de regiões intergênicas de diferentes genomas. Para quatro sequências nenhuma homologia foi encontrada, o que pode ter ocorrido é que estes primers amplificaram regiões intergênicas específicas do genoma da soja, as quais não apresentam homólogos nos genomas até então seqüenciados. Vale lembrar que nos bancos de dados, existe muita informação das regiões expressas do genoma da soja, o que leva a crer que as seqüências, para as quais não foram encontradas homologias derivam de regiões intergênicas. No entanto mesmo não ancorando sobre regiões gênicas, não se pode descartar a possibilidade de que estes primers possam amplificar regiões próximas a genes de interesse ou ainda, blocos do genoma QTLs (Quantitative Trait Loci) associado ao caráter em estudo, podendo ser promissores em estudos de mapeamentos, conforme os resultados obtidos por Henning (2007).

Cinco seqüências apresentaram homologias com seqüências de genes descritos anteriormente e depositadas nos bancos de dados avaliados (Tabela 1). O marcador Satt324 apresentou homologia com genes da enzima Diphenol oxidase, conhecida também como: O-diphenolase, Phenolase, Polyphenol oxidase, Tyrosinase, Pyrocatechol oxidase, Dopa oxidase, Catecholase, O-diphenol, Oxygen oxidoreductase, O-diphenol oxidoreductase e Catechol oxidase. Vários estudos demonstram que esta enzima está diretamente relacionada às reações de escurecimento e impermeabilidade do tegumento devido à oxidação de flavonóides (Marbach e Mayer, 1975; 
Debeaujon et al., 2003; Routaboul et al., 2006; Pourcel et al., 2007). Segundo Marbach e Mayer (1975), sementes maduras de ervilha selvagem possuem tegumentos marrons e impermeáveis à entrada de água, enquanto os tegumentos de sementes maduras de ervilha cultivada são amarelo-verdes e permeáveis à água. Estes autores constataram que, em sementes de ervilha selvagem a atividade da Diphenol oxidase aumentou continuamente durante formação das sementes, e que, o aumento da atividade desta enzima, começou quando os legumes e sementes iniciaram o processo de dessecação. Quando a quantidade de água diminuiu e o estresse hídrico tornou-se mais acentuado, foi observado o escurecimento destas sementes, como resultado de formação de quinonas e da interação entre quinonas e proteínas. Estas reações causam além do escurecimento do tegumento, a impermeabilidade a água.

TABELA 1. Marcadores microssatélites com função gênica predita pela homologia baseada no alinhamento local, de proteínas/enzimas relacionadas ao tegumento de sementes de soja.

\begin{tabular}{cccc}
\hline Microssatélite & Proteínas/Enzimas & Acesso GenBank & E-value \\
\hline Satt094 & ATPase & AK226961.1 & 0,0 \\
Satt324 & Diphenol oxidase & DQ447092.1 & $2 \mathrm{e}^{-33}$ \\
Satt277 / Satt420 & Serine threonine kinase & XM_633948.1/XM_639591.1 & $2 \mathrm{e}^{-42} / 2 \mathrm{e}^{-17}$ \\
Satt424 & PHD Zinc Finger & XM_001134472.1 & $1 \mathrm{e}^{-61}$ \\
\hline
\end{tabular}

* A seleção das homologias foi baseada na estatística de $E$-value considerando apenas valores inferiores a $1 \mathrm{e}^{-15}$.

Evidências sugerem que a oxidação de flavonóides tem função de proteção durante desenvolvimento e crescimento da planta. Por exemplo, reações de escurecimento dos tecidos são observadas durante eventos programados como dessecação de sementes (Pourcel et al., 2007). Em Arabidopsis, o escurecimento do tegumento das sementes, durante a fase de dessecação é causado através da oxidação de epicatechin e proanthocyanidins solúveis pela enzima Laccase, a qual é codificada pelo gene Transparent testA10 - TT10, que podem ligar-se a células da parede celular e criar uma barreira físico-química para proteção contra estresses (Debeaujon et al., 2003; Pourcel et al., 2005; Routaboul et al., 2006). Três enzimas principais, Laccase, Catechol oxidase ou Diphenol oxidase e Peroxidase, são conhecidas por estar envolvidas na oxidação de flavonóides. A maioria das polifenóis oxidases e peroxidases de plantas pertencem a famílias multigênicas cujos membros podem exibir redundância funcional quando simultaneamente expressos no mesmo tecido. Em Arabidopsis, o gene da família das Laccases parece ser o único membro envolvido na oxidação de flavonóides em sementes, sendo que, nenhuma das 16 outras Laccases ou Peroxidases pode compensar a perda de sua atividade no gene TT10 mutante. Durante a dessecação de sementes de ervilha, algodão e de Sida spinosa, os flavonóides acumulados nos tegumentos das sementes, são oxidados na presença de peroxidases, levando os tegumentos ao escurecimento e a impermeabilidade a água.
A formação de quinonas e polímeros insolúveis explicariam o reforço para evitar a entrada de água, ou seja, formação de tegumentos semipermeáveis (Werker et al., 1979; Egley et al., 1983). Além disto, a retirada de oxigênio pela ação da peroxidase, através da oxidação de flavonóides poderia proteger sementes da deterioração e então prolongar o período pelo qual elas podem ser armazenadas (Debeaujon et al., 2003; Bailly, 2004).

Os marcadores Satt094, Satt277, Satt420 e Satt424 apesar de estarem relacionados a enzimas envolvidas em diversas rotas metabólicas, podem também estar associados à qualidade de sementes.

Para os marcadores Satt277 e Satt420 foi encontrada homologia com genes codificadores da família de proteínas Serine/threonine protein kinases. Essas proteínas estão classificas em várias subfamílias, podendo destacar-se as Calcium dependent protein kinase (CDPK). Johnson e Chrispeels (1992) observaram que proteínas $C D P K$ foram responsáveis pela fosforilação de várias proteínas do vacúolo em sementes de feijão (Phaseolus vulgaris), e que, este processo atuava diretamente na permeabilidade dos canais das membranas. Em estudo avaliando sementes germinadas e plântulas de trigo, foi verificado que as Protein kinases (PKABA1), estavam relacionadas com respostas a desidratação, frio e tratamento com Ácido Abscísico (ABA), e que, o acúmulo destes transcritos foi observado no escutelo das sementes, durante o período de germinação (Holappa e 
Walker-Simmons, 1997). Ainda, segundo estes autores, sequências similares, que codificam para o PKABA1, foram encontradas em Arabidopsis thaliana, soja e Brassica napus. Enzimas da família Kinase são responsáveis pela maioria dos sistemas de transdução de sinais dentro das células. Diferentes proteínas dessa família atuam na transmissão de sinais para a expressão gênica, na hidrólise de lipídios, estão envolvidas na sinalização celular para ativação do sistema de defesa contra ataque de patógenos (estresse biótico) e também no sistema de sinalização do estresse causado por fatores abióticos, como o calor, frio e umidade (Shinozaki e Yamaguchi-Shinozaki, 1997; Boudsocq e Laurière, 2005). As principais funções do tegumento da soja são servir como barreira para a entrada de patógenos e/ou microorganismos (Peske e Pereira, 1983) ou ainda para a manutenção dos teores de água nas sementes. A partir do conhecimento de que as enzimas Kinase estão envolvidas nos mecanismos de sinalização de ambos os tipos de danos (umidade e patógenos), que podem comprometer a qualidade das sementes, é possível predizer que marcadores associados a genes codificadores destas enzimas são de grande interesse como marcadores moleculares para este estudo.

Para a sequência de DNA de onde foi obtido o conjunto de primers descrito como Satt094, foi detectada homologia com dois genes que codificam para as enzimas Vacuolar ATPase. Proteínas da família ATPase tem funções enzimáticas fundamentais como bombas transportadoras de prótons sendo as principais responsáveis pela redução do $\mathrm{pH}$ interno das células, alterando o potencial de membrana que é utilizado como uma fonte de energia nas funções de hidrólises das proteínas armazenadas nos tecidos celulares das sementes (Maeshima et al., 1994). As membranas do vacúolo central contêm dois tipos distintos de bombas de prótons, $\mathrm{H}^{+}$-ATPase e $\mathrm{H}^{+}$-PPase (Sze, 1985; Rea e Poole, 1986; Hedrich e Schroeder, 1989; Taiz, 1992). Estas bombas de prótons diminuem o $\mathrm{pH}$ interno e o potencial de membrana, o que é utilizado como uma fonte de energia nos sistemas de transporte secundários de membrana vacuolar. Entre as proteínas de membrana do vacúolo central, estas duas bombas de prótons foram bem caracterizadas em suas estruturas moleculares e propriedades enzimáticas (Gogarten et al., 1989; Maeshima e Yoshida, 1989; Matsuura-Endo et al., 1990; Rea et al., 1992; Sze et al., 1992). Nos processos de germinação de sementes, a acidificação do vacúolo pode ser necessária em funções biológicas como a hidrólise de proteínas armazenadas na semente. Alguns estudos mostraram o aumento na expressão de genes que codificam para proteínas da família ATPase em sementes de tomate, responsivas ao tratamento com ácido giberélico, este aumento foi detectado nas sementes antes de aparecimento de radícula, evidenciando que a expressão destes genes é importante nos primeiros estágios de germinação das sementes (Cooley et al., 1999). O mesmo fato foi observado em sementes de Fagus sylvatica tratadas com ácido giberélico, para a superação de dormência (Lorenzo et al., 2002). Esses resultados sugerem que o ácido giberélico funciona como ativador na expressão dessas enzimas, sendo esta correlacionada ao aumento percentual na germinação dessas sementes. Geralmente as modificações no tegumento relacionadas com o aumentam da resistência física das sementes também estão relacionadas à dormência das sementes, pois, impedem a entrada de agentes que promovem a germinação, tal como oxigênio e água, ou até mesmo de substâncias inibidoras como ácido de abscíssico ou gás carbônico (Benech-Arnold et al., 2006).

As sequências do marcador Satt424 mostrou homologia com genes que codificam para proteínas PHD Zinc Finger. Proteínas com domínios Zinc finger representam uma importante classe de fatores de transcrição para diferentes genes em eucariotos (Yoshioka et al., 2001). Proteínas descritas como PHD Zinc Finger (Plant Homeodomain) são relatadas como responsáveis em ligar-se a histonas (H3) atuando como efetores da transcrição de diferentes genes (Halbach et al., 2000). Grande parte do sistema de transdução de sinais celulares é mediada por um complexo sistema de dependências encontradas na membrana das células, onde moléculas de Phosphatidylinositol phosphates são responsáveis pelo controle de várias sequências de sinais envolvidas em diversos aspectos fisiológicos, incluindo a proliferação e morte celular, organização do citoesqueleto, tráfico intracelular e metabolismo, além de interagir como sinalizador para rotas de mensageiros secundários como as proteínas Calcium Kinase, Phosphorus Kinase, Inositol 1,4,5-triphosphate e Diacylglycerol. Parte da transdução de sinais em resposta ao estresse abiótico e biótico, como diferentes taxas no acumulo de ABA e fechamento de estômatos passa por esta rota metabólica (Shinozaki e Yamaguchi-Shinozaki, 1997). Uma interação entre proteínas PHD Zinc Finger e a Phospatidil inositol foi descrita por Kaadige e Ayer (2006), o que, possivelmente explica a relação deste marcador com diferenças fenotípicas em sementes. Confirmações experimentais para esta associação foram verificadas em plantas de Arabidopsis com diferentes respostas na dormência das sementes mediada por $\mathrm{ABA}$, onde foram observadas diferentes taxas de expressão de proteínas da família Zinc Finger (He e Gan, 2004). Em genótipos mutantes de arroz para a produção de $\mathrm{ABA}$, submetidos ao 
estresse por desidratação, também foi verificada alteração nas taxas de síntese de genes da família Zinc Finger, incluindo um gene de PHD zinc finger (Oh et al., 2005). Por este gene estar descrito como envolvido na transdução de sinais da rota metabólica do $\mathrm{ABA}$, pode ser considerado como um importante marcador para o estudo em genótipos com diferentes características de semente.

\section{CONCLUSÕES}

Os resultados desta análise indicam como promissor o uso dos marcadores Satt094, Satt277, Satt324, Satt420 e Satt 424 para estudos relacionados ao tegumento e à qualidade de sementes de soja.

A estratégia da mineração de marcadores moleculares a partir da integração in silico de seqüências de marcadores moleculares ainda anônimos, bancos de dados genômicos e bancos contendo seqüências com descrições funcionais dos genes demonstra ser promissora, pois, possibilita prever as funções para estes genes e verificar a associação destes com rotas bioquímicas e metabólicas responsáveis pelas características que se deseja analisar em rotinas in vitro.

O uso desta estratégia, integrando conhecimentos sobre um modelo biológico de uma ou mais espécies, pressuposições bioquímicas e fisiológicas dos caracteres a serem estudados e a associação destes com informações sobre marcadores moleculares e dados da genômica funcional e estrutural é a principal aplicação prática da bioinformática no contexto das ciências agronômicas, pois possibilita prever antecipadamente quais marcadores moleculares são os melhores candidatos para diferentes aplicações, resultando na redução de custos e tempo.

\section{AGRADECIMENTOS}

Os autores agradecem a CAPES (Coordenação de Aperfeiçoamento de Pessoal de Nível Superior), ao CNPq (Conselho Nacional de Desenvolvimento Científico e Tecnológico) e a FAPERGS (Fundação de Amparo à Pesquisa do Rio Grande do Sul), pelo apoio financeiro concedido aos autores em diversas instâncias durante o desenvolvimento desse trabalho.

\section{REFERÊNCIAS}

ALTSCHUL, S.F.; GISH W.; MILLER, W.; MYERS E.W.; LIPMAN, D.J. Basic local alignment search tool. Journal of Molecular Biology, .215, n.3, p.403-410, 1990.
BAILLY, C. Active oxygen species and antioxidants in seed biology. Seed Science Research, v.14, n.2, p.93-107, 2004.

BENECH-ARNOLD, R.L.; GUALANO, N.; LEYMARIE, J.; CÔME, D.; CORBINEAU, F. Hypoxia interferes with ABA metabolism and increases ABA sensitivity in embryos of dormant barley grains. Journal of Experimental Botany, v.57, n.6, p.1423-1430, 2006.

BOUDSOCQ, M.; LAURIÈRE, C. Osmotic signaling in plants: multiple pathways mediated by emerging kinase families. Plant Physiology, v.138, n.3, p.1185-1194, 2005.

BUSO, G.S.C.; CIAMPI, A.Y.; MORETZSOHN, M.C.; AMARAL, Z.P.S.; BRONDANI, R.V. Marcadores Microssatélites em Espécies Vegetais. Biotecnologia Ciência e Desenvolvimento, v.7, p.46-50, 2003.

COOLEY, M.B.; YANG, H.; DAHAL, P.; MELLA, R.A.; DOWNIE, A.B.; HAIGH, A.M.; BRADFORD, K.J. Vacuolar $\mathrm{H}(+)$-ATPase is expressed in response to gibberellin during tomato seed germination. Plant Physiology, v.121, n.4, p.1339-1348, 1999.

DEBEAUJON, I.; NESI, N.; PEREZ, P.; DEVIC, M.; GRANDJEAN, O.; CABOCHE, M.; LEPINIEC, L. Proanthocyanidin-accumulating cells in Arabidopsis testa: regulation of differentiation and role in seed development. Plant Cell, v.15, n.11, p.2514-2531, 2003.

EGLEY, G.H.; PAUL, R.N.; VAUGAN, K.C.; DUKE, S.H. Role of peroxidase in the development of water impermeable seed coat in Sida spinosa L. Planta, v.157, n.3, p.224-232, 1983.

GOGARTEN, J.P.; KIBAK, H.; DITTRICH, P.; TAIZ, L.; BOWMAN, E.J.; BOWMAN, B.J.; MANOLSON, M.F.; POOLE, R.J.; DATE, T.; OSHIMA, T. Evolution of the vacuolar $\mathrm{H}+$-ATPase: implications for the origin of eukaryotes. Proceedings of the National Academy of Sciences, v.86, n.17, p.6661-6665, 1989.

HALBACH, T.; SCHEER, N.; WERR, W. Transcriptional activation by the PHD finger is inhibited through an adjacent leucine zipper that binds 14-3-3 proteins. Nucleic Acids Research, v.28, n.18, p.3542-3550, 2000.

HE, Y.; GAN, S. A novel zinc-finger protein with a prolinerich domain mediates ABA-regulated seed dormancy in Arabidopsis. Plant Molecular Biology, v.54, n.1, p.1-9, 2004.

HEDRICH, R.; SCHROEDER, J.I. The physiology of ion channels and electrogenic pumps in higher plants. Annual Review of Plant Physiology, v.40, p.539-569, 1989.

HENNING, F.A. Mapeamento de caracteres do tegumento 
da semente de soja e análise in silico dos marcadores microssatélites. Pelotas, 2007, 58 f. Dissertação (Mestrado), Universidade Federal de Pelotas.

HOLAPPA, L.D.; WALKER-SIMMONS, M.K. The wheat protein kinase gene, TaPK3, of the PKABA1 subfamily is differentially regulated in greening wheat seedlings. Plant Molecular Biology, v.33, n.5, p.935-941, 1997.

JOHNSON, K.D.; CHRISPEELS, M.J. Tonoplast-Bound Protein Kinase Phosphorylates Tonoplast Intrinsic Protein. Plant Physiology, v.100, n.4, p.1787-1795, 1992.

KAADIGE, M.R.; AYER, D.E. The polybasic region that follows the plant homeodomain zinc finger 1 of Pf1 is necessary and sufficient for specific phosphoinositide binding. Journal of Biological Chemistry, v.281, n.39, p.28831-28836, 2006.

LIANG, C.; JAISWAL, P.; HEBBARD, C.; AVRAHAM, S.; BUCKLER, E.S.; CASSTEVENS, T.; HURWITZ, B.; MCCOUCH, S.; NI, J.; PUJAR, A.; RAVENSCROFT, D.; REN, L.; SPOONER, W.; TECLE, I.; THOMASON, J.; TUNG, C.W.; WEI, X.; YAP, I.; YOUENS-CLARK, K.; WARE, D.; STEIN, L. Gramene: a growing plant comparative genomics resource. Nucleic Acids Research, 2007 Nov 4; [Epub ahead of print] PMID: 17984077 [PubMed - as supplied by publisher]. (Gramene Reference ID 11653 )

LITT, M.; LUTTY, J.A. A hypervariable microsatellite revealed by in vitro amplification of a dinucleotide repeat within the cardiac muscle actin gene. American Journal of Human Genetics, v.44, n.3, p.397-401, 1989.

LORENZO, O.; NICOLÁS, C.; NICOLÁS, G.; RODRÍGUEZ, D. GA(3)-induced expression of a new functional AAA-ATPase (FsA1) is correlated with the onset of germination in Fagus sylvatica L. seeds (beechnuts). Plant and Cell Physiology, v.43, n.1, p.27-34, 2002.

MAESHIMA, M.; YOSHIDA, S. Purification and properties of vacuolar membrane proton-translocating inorganic pyrophosphatase from mung bean. Journal of Biological Chemistry, v.264, n.33, p.20068-20073, 1989.

MAESHIMA, M.; HARA-NISHIMURA, I.; TAKEUCHI, Y.; NISHIMURA, M. Accumulation of Vacuolar $\mathrm{H}+-$ Pyrophosphatase and $\mathrm{H}+$-ATPase during Reformation of the Central Vacuole in Germinating Pumpkin Seeds. Plant Physiology, v.106, n.1, p.61-69, 1994.

MARBACH, I.; MAYER, A.M. Changes in Catechol Oxidase and Permeability to Water in Seed Coats of Pisum elatius during Seed Development and Maturation. Plant Physiology, v.56, n.1, p.93-96, 1975.
MATSUURA-ENDO, C.; MAESHIMA, M.; YOSHIDA, S. Subunit composition of vacuolar membrane $\mathrm{H}(+)$-ATPase from mung bean. European Journal of Biochemistry, v.187, n.3, p.745-751, 1990.

MATTHEWS, D.E.; CAROLLO, V.L.; LAZO, G.R.; ANDERSON, O.D. GrainGenes, the genome database for small-grain crops. Nucleic Acids Research, v.31, n.1, p.183-186, 2003.

MORGANTE, M.; OLIVIERI, A.M. PCR-amplified microsatellites as markers in plant genetics. The Plant Journal, v.3, n.1, p.175-182, 1993.

OH, S.J.; SONG, S.I.; KIM, Y.S.; JANG, H.J.; KIM, S.Y.; KIM, M.; KIM, Y.K.; NAHM, B.H.; KIM, J.K. Arabidopsis $\mathrm{CBF} 3 / \mathrm{DREB} 1 \mathrm{~A}$ and $\mathrm{ABF} 3$ in transgenic rice increased tolerance to abiotic stress without stunting growth. Plant Physiology, v.138, n.1, p.341-351, 2005.

PESKE, S.T.; PEREIRA, L.A.G. Tegumento da semente de soja. Tecnologia de sementes, v.6, p. 23-34, 1983.

PONCET, V.; HAMON P.; SAUVAGE, De SAINT MARC M-B.; BERNARD, T.; HAMON, S.; NOIROT, M. Base composition of Coffea AFLP sequences and their conservation within the genus. Journal of Heredity, v.96, n.1, p.59-65. 2005.

POURCEL, L.; ROUTABOUL, J.M.; CHEYNIER, V.; LEPINIEC, L.; DEBEAUJON, I. Flavonoid oxidation in plants: from biochemical properties to physiological functions. Trends Plant Science, v.12, n.1, p.29-36, 2007.

POURCEL, L.; ROUTABOUL, J.M.; KERHOAS, L.; CABOCHE, M.; LEPINIEC, L.; DEBEAUJON, I. TRANSPARENT TESTA10 encodes a laccase-like enzyme involved in oxidative polymerization of flavonoids in Arabidopsis seed coat. Plant Cell, v.17, n.11 p.2966-2980, 2005.

REA, P.A.; KIM, Y.; SARAFIAN, V.; POOLE, R.J.; DAVIES, J.M.; SANDERS, D. Vacuolar H(+)-translocating pyrophosphatases: a new category of ion translocase. Trends in Biochemical Sciences, v.17, n.9, p.348-353, 1992.

REA, P.A.; POOLE, R.J. Chromatographic Resolution of H-Translocating Pyrophosphatase from H-Translocating ATPase of Higher Plant Tonoplast. Plant Physiology, v.81, n.1, p.126-129, 1986.

ROUTABOUL, J.M.; KERHOAS, L.; DEBEAUJON, I.; POURCEL, L.; CABOCHE, M.; EINHORN, J.; LEPINIEC, L. Flavonoid diversity and biosynthesis in seed of Arabidopsis thaliana. Planta, v.224, n.1, p.96-107, 2006. 
SHINOZAKI, K.; YAMAGUCHI-SHINOZAKI, K. Gene Expression and Signal Transduction in Water-Stress Response. Plant Physiology, v.115, n.2, p.327-334, 1997.

SZE, H. H+-translocating ATPases: advances using membrane vesicles. Annual Review of Plant Physiology, v.36, p.175-206, 1985.

SZE, H.; WARD, J.M.; LAI, S.; PERERA, I.I. Vacuolartype $\mathrm{h}+$-translocating ATPases in plant endomembranes: subunit organization and multigene families. The Journal of Experimental Biology. v.172, n.1, p.123-135, 1992.

TAIZ, L. The plant vacuole. The Journal of Experimental Biology, v.172, n.1, p.113-122, 1992.
TAUTZ, D. Hypervariability of simple sequences as a general source for polymorphic DNA markers. Nucleic Acids Research, v.17, n.16, p.6463-6471, 1989.

WERKER, E.; MARBACH, I.; MAYER, A.M. Relation between the anatomy of the testa, water permeability and the presence of phenolics in the genus Pisum. Annals of Botany, v.43, p.765-771, 1979.

YOSHIOKA, K.; FUKUSHIMA, S.; YAMAZAKI, T.; YOSHIDA, M.; TAKATSUJI, $H$. The plant zinc finger protein ZPT2-2 has a unique mode of DNA interaction. Journal of Biological Chemistry, v.276, n.38, p.35802$35807,2001$. 